\title{
Comportamento de vacas leiteiras submetidas a um manejo aversivo
}

\author{
Maria José Hötzel* \\ Carla Christina de Miranda Gomes \\ Luiz Carlos Pinheiro Machado Filho \\ Laboratório de Etologia Aplicada \\ Departamento de Zootecnia e Desenvolvimento Rural \\ Universidade Federal de Santa Catarina \\ Rodovia Admar Gonzaga, 1346, CEP 88.034-001, Florianópolis - SC, Brasil \\ *Autor para correspondência \\ mjhotzel@cca.ufsc.br
}

Submetido em 24/03/2008

Aceito para publicação em 14/09/2008

\section{Resumo}

Em pequenas propriedades leiteiras, devido à falta de instalações adequadas para o manejo, as vacas podem ser contidas e submetidas à inspeção ou tratamento veterinário no seu ambiente de ordenha, o que, por sua vez, pode influenciar o comportamento dos animais, perturbando a rotina de manejo. Sete vacas leiteiras, mantidas em sistema intensivo de pastoreio rotativo e ordenhadas duas vezes por dia por dois manejadores, foram expostas a um minucioso exame clínico, durante três dias consecutivos. Dados comportamentais antes e depois do procedimento foram analisados através de uma análise de variância. O comportamento das vacas durante o procedimento indicou forte aversividade. O tratamento não influenciou a distância de fuga mantida em relação ao veterinário ou a uma pessoa desconhecida pelas vacas, avaliadas antes e após o procedimento veterinário (veterinário: antes $=1,2 \pm 0,1$; após $0,8 \pm 0,2$; desconhecido: $1,0 \pm 0,2$ após $1,2 \pm 0,2 ; \mathrm{p}=0,3$ ), nem o número de interações agonísticas no grupo, observadas antes $(7,1 \pm 2)$ e após $(11,5 \pm 3)$ o procedimento $(p=0,3)$, ou um escore de reatividade atribuído a cada animal $(p=0,2)$. Estes resultados não permitem concluir que a aplicação repetida e inevitável de procedimentos veterinários no ambiente da ordenha influencia o comportamento das vacas durante a ordenha ou a sua reatividade ao homem.

Unitermos: gado de leite, relação humano-animal, distância de fuga, etologia, bem-estar animal

\section{Abstract}

Behaviour of dairy cows subjected to an aversive veterinary procedure. On small dairy farms that lack appropriate handling facilities, cows can be restrained and subjected to veterinary inspection or treatment in their milking environment, which in turn might influence the behaviour of the animals, disrupting routine management. A group of seven dairy cows kept on an intensive rotational pasture system and machine milked twice a day by two familiar handlers were exposed to a thorough clinical examination for three consecutive days. Behavioural data before and after the procedure were analyzed by ANOVA. The behaviour of all the cows during the procedure indicated strong aversiveness. Treatment did not influence the flight distance (metres) kept from the veterinarian or from a person unknown by the cows, assessed before and after the procedure (veterinarian: 
before $=1.2 \pm 0.1$; after $0.8 \pm 0.2$; unknown: $1.0 \pm 0.2$ after $1.2 \pm 0.2 ; \mathrm{p}=0.3)$, nor did it affect the number of agonistic interactions within the group observed before $(7.1 \pm 2)$ and after $(11.5 \pm 3)$ the procedure $(p=0.3)$, or reactivity score $(\mathrm{p}=0.2)$. These results do not support the conclusion that the repeated application of unavoidable aversive veterinary procedures in the milking environment will influence the behaviour of cows during milking or their reactivity to humans.

Key words: dairy cattle, human-animal relationship, flight distance, applied ethology, animal welfare

\section{Introdução}

Vários procedimentos do manejo de rotina na criação de bovinos de leite podem envolver comportamentos humanos que são aversivos para os animais, como berros, tapas e choques elétricos (Pajor et al., 2003). Em trabalhos anteriores foi demonstrado que vacas aprendem a reconhecer pessoas que as tratam aversivamente, passando a manter-lhes uma maior distância de fuga (Rushen et al., 1999a; Munksgaard et al., 2001; Yunes, 2001; Hötzel et al., 2005). Alguns desses trabalhos sugerem que isso pode interferir com a produção leiteira, através do aumento do leite residual (Rushen et al., 1999a; Breuer et al., 2000). A aplicação de tratamentos de natureza aversiva também pode ter conseqüências negativas no manejo, ao gerar um comportamento de evitação nos animais confrontados com humanos que consideram aversivos; isso, por sua vez, pode reforçar a atitude negativa dos manejadores em relação aos animais, levando-os a manter o seu comportamento aversivo para com os animais, num indesejável processo de retro-alimentação (Hemsworth e Coleman, 1998). Assim como as experiências vivenciadas pelos animais podem ser associadas à pessoa que os tratou aversivamente (Munksgaard et al., 1997; Rushen et al., 1999b) também podem ser generalizadas a outras pessoas (Hemsworth et al., 1994 e 1996; Munksgaard et al., 1999); além disso, existe evidência de que os animais também podem associar o tratamento aversivo ao local onde o mesmo foi realizado (de Passille et al., 1996).

A realização de procedimentos veterinários é indispensável para a manutenção da sanidade animal e faz parte do manejo reprodutivo do rebanho leiteiro. Considera-se que vários desses procedimentos são aversivos para os animais, especialmente quando causam desconforto ou dor, a exemplo da palpação retal (Nakao et al., 1994) e do corte dos cascos (Lewis e Hurnik, 1998). Ao contrário de maus tratos recebidos durante o manejo diário, que geralmente acontecem repetidamente e podem ser evitados através da educação dos manejadores (Hemsworth e Coleman, 1998), procedimentos veterinários acontecem esporadicamente, mas não podem ser evitados.

Em pequenas propriedades, por falta de uma infraestrutura apropriada para a realização de procedimentos veterinários, os animais são freqüentemente contidos e sujeitos a tratamentos veterinários no seu ambiente de ordenha. Conseqüentemente, procedimentos veterinários aversivos que necessitem ser aplicados repetidamente podem ser associados à pessoa que o realizou, o que pode dificultar a realização de procedimentos subseqüentes; se essa experiência for generalizada a outras pessoas, isso pode causar problemas no manejo de rotina, especialmente na condução dos animais entre ordenhas, entre o pasto e a sala de ordenha, e dentro da mesma. Adicionalmente, se a aversividade do procedimento for associada ao local onde o mesmo foi realizado, pode também dificultar o manejo dos animais nesse local. O objetivo do presente estudo foi verificar essas questões.

\section{Material e Métodos}

O presente estudo foi desenvolvido na Unidade de Gado de Leite do Centro de Treinamento da EPAGRI (Empresa de Pesquisa Agropecuária e Extensão Rural de Santa Catarina), em Florianópolis, SC, entre os dias 6 e 21 de agosto de 2007.

No estudo foram utilizadas sete vacas em lactação, com produção leiteira média de $15 \mathrm{l} / \mathrm{vaca} / \mathrm{dia}$. A rotina dos animais foi mantida durante o experimento. Os animais eram ordenhados diariamente com ordenhadeira mecânica em dois períodos, às $6: 00 \mathrm{~h}$ e $16: 30 \mathrm{~h}$. No período entre ordenhas as vacas permaneciam juntas em 
piquetes em sistema rotativo. Por aproximadamente meia hora antes e após a ordenha, os animais permaneciam em um curral localizado ao lado da sala de ordenha, com acesso a água e sal mineral, mas sem alimento. Durante a ordenha os animais recebiam alimento concentrado no cocho individualmente, de acordo com a fase de lactação, à base de farelo de trigo e grãos.

As vacas foram identificadas a partir de uma marcação feita com tinta atóxica, combinando número e cor, no primeiro dia do experimento. O padrão de manchas (foto e desenho) e a numeração dos brincos também auxiliaram na identificação de cada vaca.

O comportamento dos animais durante o procedimento veterinário foi observado a fim de verificar o grau de aversividade do tratamento. Foram considerados sinais de aversividade, como o afastamento do animal em resposta ao movimento do veterinário, aumento da movimentação de patas, orelha, cauda, cabeça, exposição da esclera (olho arregalado), expansão da caixa torácica, bufadas, ocorrência de defecação e urina, cabeçadas e coices.

Um exame veterinário foi realizado em três dias consecutivos, antes da ordenha da tarde, em cada vaca individualmente. Para o exame, a vaca era contida em uma baia individual de ordenha, que consistia em duas paredes laterais de madeira de $1,4 \mathrm{~m}$ de altura, um cocho na frente e uma corrente atrás. O tratamento, que durava entre 15 e $20 \mathrm{~min}$, iniciava pela aproximação do veterinário, pela baia vizinha, lateralmente à vaca. $\mathrm{O}$ veterinário dava início ao exame clínico com a palpação de linfonodos cervicais e inguinais, aferição de frequiências cardíaca e respiratória com auxílio de estetoscópio, seguindo da aferição da temperatura por via retal, palpação e inspeção do úbere, percussão torácica com auxílio de martelo e placa de percussão, inspeção da esclera, inspeção da boca com exposição da língua e inspeção e palpação retal. Um dos manejadores dos animais permaneceu no local, nos três dias, durante a execução do exame físico e conduziu a saída e entrada dos animais no pasto, na sala de espera da ordenha e nas baias.

Foram aplicados testes de distância de fuga no pasto. O teste foi repetido nos dois dias anteriores e nos dois dias posteriores à aplicação do tratamento, por duas pessoas - o veterinário e uma pessoa com quem os animais somente tiveram contato nesses dias (doravante denominada neutra). A ordem da realização dos testes foi sorteada e a partir de então, alternada. O teste de distância de fuga consiste em medir a distância máxima, em metros, que o animal permite uma pessoa se aproximar, conforme descrito em Hötzel et al. (2005). O veterinário vestia sempre macacão cinza e botas pretas, tanto durante a realização do exame físico quanto para a aplicação dos testes de distância de fuga.

Nos mesmos dias, o comportamento de cada vaca foi avaliado durante a ordenha. Desde a entrada do animal na sala de ordenha, foi observada a resposta de cada vaca aos comandos do ordenhador, a sua resposta à aproximação do ordenhador para amarrar as patas e colocar as ordenhadeiras. Também foram observadas as respostas comportamentais como movimentação de orelhas, cabeça, patas e cauda, e ocorrência de defecação e urina. A seguir, observaram-se os mesmos comportamentos em relação a uma terceira pessoa que se aproximava dos animais, tocando-os na paleta, nos flancos e no úbere. A partir desses dados, um escore de reatividade foi atribuído a cada animal (Tabela 1), usando metodologia adaptada de Honorato (2006).

Também foram registradas as interações agonísticas ocorridas entre as vacas, durante um período de duas horas, em duas ocasiões antes e duas ocasiões após o tratamento.

Os dados de distância de fuga foram comparados através de uma análise de variância simples, considerando os fatores fixos período - antes e após o tratamento e tratador - veterinário ou neutro - e as vacas como repetição. Para os dados de reatividade e interações agonísticas foi considerado somente o fator período (SuperANOVA, Abacus Concepts, Inc., USA).

O protocolo experimental foi aprovado pelo Comitê de Experimentação Animal da Universidade Federal de Santa Catarina (CEUA), que atende as recomendações do Colégio Brasileiro em Experimentação Animal. 
TABELA 1: Descrição da avaliação do comportamento das vacas na sala de ordenha, para atribuição do escore de reatividade.

\begin{tabular}{|c|c|c|}
\hline Escore & Temperamento & Comportamento \\
\hline 0 & Dócil & $\begin{array}{l}\text { Encaminha-se calmamente para a sua baia } \\
\text { Movimenta-se pouco } \\
\text { Posicionado facilmente para observação } \\
\text { Não se perturba quando manipulado, observado ou durante a ordenha, continua a fazer o } \\
\text { que estava fazendo anteriormente (ex.: comendo) }\end{array}$ \\
\hline 0,5 & Levemente Agitado & $\begin{array}{l}\text { Comportamento vigilante: parado, olhar fixo e movimenta orelha em direção ao ruído/mo- } \\
\text { vimento/pessoa } \\
\text { Para de fazer o que estava fazendo (ex.: comendo) }\end{array}$ \\
\hline 1 & Agitado & $\begin{array}{l}\text { Mostra certa resistência ao encaminhar-se para a baia } \\
\text { Movimentos de cauda, cabeça, patas e abertura das narinas durante a manipulação, obser- } \\
\text { vação e ordenha } \\
\text { Urina, defeca }\end{array}$ \\
\hline 1,5 & Muito Agitado & $\begin{array}{l}\text { Empaca durante a condução para a sala de ordenha } \\
\text { Resistência ao toque } \\
\text { Urina, defeca }\end{array}$ \\
\hline 2 & Violento & $\begin{array}{l}\text { Entra na baia após ser forçada } \\
\text { Não permanece muito tempo na mesma posição, } \\
\text { movendo-se continuamente, movimenta bastante a cauda, sapateia durante manipulação, } \\
\text { observação e ordenha } \\
\text { Movimentos respiratórios marcantes (tórax e narinas) } \\
\text { Urina, defeca } \\
\text { Reação com violência ao toque (cabeçada, coice, mordida) }\end{array}$ \\
\hline
\end{tabular}

\section{Resultados e Discussão}

Não houve efeito do tratador nem do dia em que os animais foram testados, ou interações, na distância de fuga dos animais (Figura 1). Ou seja, o tratamento veterinário não se refletiu em um aumento na distância de fuga das vacas em relação ao veterinário que aplicou o tratamento, nem a outra pessoa, desconhecida pelos animais e que não participou do tratamento. $\mathrm{O}$ aumento da distância de fuga em vacas, observado após tratamentos aversivos que simulam maus tratos, é interpretado como manifestação de medo nos animais (Rushen et al., 1999a; Hötzel et al., 2005). O comportamento observado nos animais neste estudo sugerem que o tratamento realizado não levou os animais a desenvolverem medo das pessoas envolvidas.

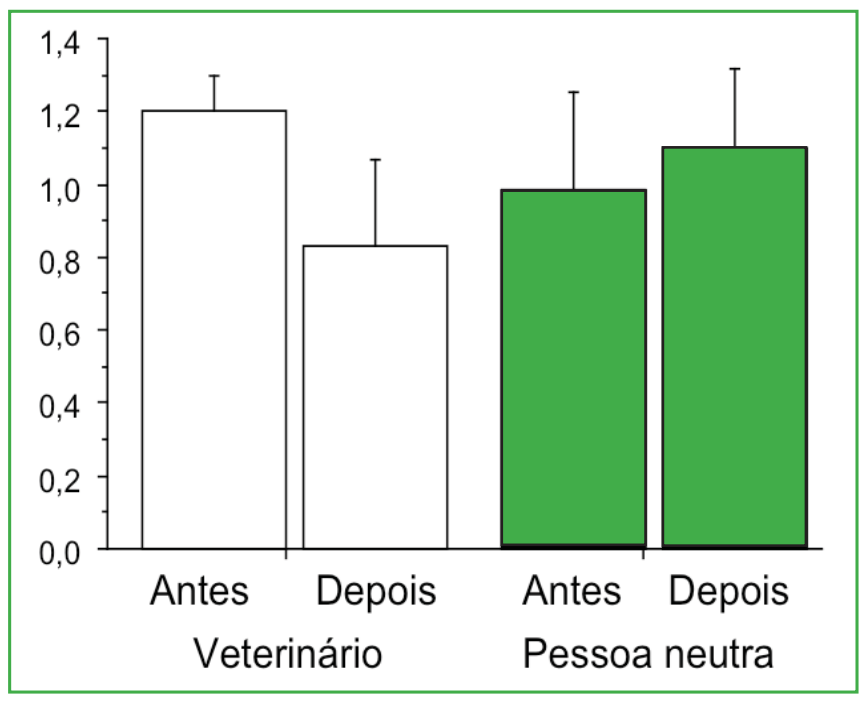

FIGURA 1: Distância de fuga das vacas em relação ao veterinário e a uma pessoa desconhecida (neutra), antes e após três dias consecutivos da aplicação de um procedimento veterinário aversivo. 
Não houve diferenças entre antes e após o procedimento veterinário, no escore de reatividade (antes $=0,3 \pm 0,2$ e depois $0,6 \pm 0,1 ; \mathrm{p}=0,2)$, ou o número de interações agonísticas ocorridas entre os animais (antes $=7,1 \pm 2$ e depois $=11,5 \pm 3 ; p=0,3)$. Diferenças nessas variáveis poderiam sugerir que houve generalização do estímulo aversivo do tratamento ao local onde o mesmo foi realizado, ou um aumento no estresse social do grupo, o que não parece ter ocorrido neste estudo.

Durante a aplicação do tratamento, todos os animais apresentaram comportamentos que sugerem aversividade, evitando o veterinário, retirando a cabeça durante a contenção e tentativa de exposição da língua, arqueando o dorso e recuando. Outros comportamentos incluíram a movimentação contínua de patas, orelha, cauda e cabeça, exposição da esclera (olho arregalado), expansão da caixa torácica, bufadas, defecação e urina, cabeçadas e coices, que ocorreram durante todo o procedimento veterinário e principalmente durante a inspeção da esclera e da boca. Ou seja, os resultados não podem ser explicados pelo fato do tratamento veterinário não ter sido suficientemente aversivo para alterar o comportamento das vacas.

$\mathrm{O}$ fato de o teste de distancia de fuga ter sido realizado em um local diferente de onde os animais receberam o tratamento aversivo tampouco pode explicar os resultados, já que estudos anteriores mostram que vacas podem reconhecer tratadores aversivos quando testados em local diferente de onde ocorreu o tratamento (de Passille et al., 1996; Pinheiro Machado $\mathrm{F}^{0}$ et al., 2001), mesmo após vários meses sem haver contato entre os animais e a pessoa que os tratou aversivamente (Hötzel et al., 2005).

Por outro lado, os três dias do tratamento podem ter sido insuficientes para permitir que os animais aprendessem a reconhecer o veterinário. De fato, os trabalhos que mostram que vacas leiteiras aprendem a reconhecer indivíduos pela qualidade dos tratamentos que deles recebem, mantendo maiores distâncias de fuga das pessoas aversivas, repetiram os tratamentos entre sete e dezenove vezes (Rushen et al., 1999a; Pinheiro Machado $F^{\circ}$ et al., 2001; Hötzel et al., 2005). Rushen et al. (1999a), por exemplo, encontraram um aumento na distância de fuga de vacas, após dez dias seguidos de um tratamento que visou simular maus tratos, como tapas e berros, mas não cinco dias após o início do tratamento. Isso sugere que a duração do tratamento neste estudo, de apenas três dias, pode explicar a ausência de alterações no comportamento dos animais. Entretanto, ao passo que maus tratos geralmente se repetem diariamente na rotina do manejo, a administração de procedimentos veterinários é normalmente pontual e de curta duração, como realizado no experimento; portanto, a duração do tratamento neste estudo pode ser considerada suficiente para responder às questões colocadas.

Outro fator que pode ter contribuído para os resultados deste estudo é a qualidade geral do tratamento que os animais recebiam na rotina, anteriormente ao tratamento. As vacas utilizadas no presente estudo eram intensamente manejadas, tanto pela rotina da ordenha como por serem criadas em um sistema de pastoreio rotativo, que se caracteriza pela troca diária de piquetes. Os baixos escores de reatividade dos animais, entre $0 \mathrm{e}$ 1 , sugerem que a qualidade dos tratamentos durante a rotina era positiva ou neutra. Assim como experiências aversivas ocorridas anteriormente podem aumentar a reatividade dos animais, como é o caso da condução de bovinos em centros de manejo (Lewis e Hurnik, 1998), por outro lado, o contato positivo entre os manejadores e os animais durante a rotina diminui as reações de estresse e a reatividade dos animais às pessoas e facilita o manejo em geral (Hargreaves e Hutson, 1990; Boivin et al., 1992; Hemsworth et al., 1994). A presença, durante a execução do exame veterinário, do ordenhador conhecido pelos animais, também pode ter amenizado o efeito aversivo do tratamento, como sugerido no estudo de Waiblinger et al. (2004), que mostrou que o estresse ocasionado por procedimentos veterinários envolvendo palpação retal e introdução de sonda de inseminação pode ser reduzido por um período prévio de tratamento gentil e pela presença de pessoas conhecidas.

Conforme sugerido por Lewis e Hurnik (1998) procedimentos aversivos, quando aplicados de forma esporádica, não parecem ter efeitos subseqüentes no comportamento dos animais. Assim, a realização de procedimentos veterinários da natureza dos que foram aplicados neste estudo, apesar de aparentemente ser aversiva para vacas leiteiras, não influencia o 
comportamento desses animais após a sua realização. Quando aplicados em animais que recebem manejo freqüente, de natureza neutra ou positiva, o aumento na reatividade dos animais não ultrapassa o momento em que o tratamento foi aplicado, não é generalizado a outras pessoas desconhecidas e que não participaram do procedimento, nem é associado negativamente ao local onde ocorreram. Possivelmente, a presença de pessoas conhecidas pelos animais durante o procedimento contribui para diminuir os seus efeitos negativos no comportamento desses animais.

\section{Agradecimentos}

À Epagri, por permitir a realização do estudo em suas instalações, e aos seus funcionários, que colaboraram nas atividades de rotina. Às estagiárias do Laboratório de Etologia Aplicada - Carolina Gasparin, estudante da Universidade Federal do Paraná, e Lídia A. Teodoro.

\section{Referências}

Boivin, X.; Boissy, A.; Nowak, R.; Henry, C.; Tournadre, H.; Le Neindre, P. 2002. Maternal presence limits the effects of early bottle feeding and petting on lambs' socialisation to the stockperson. Applied Animal Behaviour Science, 77: 311-328.

Breuer, K.; Hemsworth, P. H.; Barnett, J. L.; Matthews, L. R; Coleman, G. J. 2000. Behavioural response to humans and the productivity of commercial dairy cows. Applied Animal Behaviour Science, 66: 273-288.

de Passille, A. M.; Rushen, J.; Ladewig, J.; Petherick, C. 1996. Dairy calves discrimination of people based on previous handling. Journal of Animal Science, 74: 969-975.

Hargreaves, A. L.; Hutson, G. D. 1990. The effect of gentling on heart rate, flight distance and aversion of sheep to a handling procedure. Applied Animal Behaviour Science, 26: 243-252.

Hemsworth, P. H.; Coleman, G. J. 1998. Human-livestock interactions: The stockperson and the productivity and welfare of intensively-farmed animals. $\mathrm{CAB}$ International, Oxon, UK, $152 \mathrm{pp}$.

Hemsworth, P. H.; Coleman, G. J.; Cox, M.; Barnett, J. L. 1994. Stimulus- generalization - the inability of pigs to discriminate between humans on the basis of their previous handling experience. Applied Animal Behaviour Science, 40: 129-142.
Hemsworth, P. H.; Verge, J.; Coleman, G. J. 1996. Conditioned approach- avoidance responses to humans: the ability of pigs to associate feeding and aversive social experiences in the presence of humans with humans. Applied Animal Behaviour Science, 50 (1): 71-82.

Honorato, L. A. 2006. A interação humano-animal e o uso de homeopatia em bovinos de leite. Dissertação de Mestrado, Universidade Federal de Santa Catarina, Brasil, 120pp.

Hötzel, M. J.; Machado Filho, L. C. P.; Yunes, M. C.; Silveira, M. C. A. C. 2005. Influência de um ordenhador aversivo sobre a produção leiteira de vacas da raça holandesa. Revista Brasileira de Zootecnia, 34 (4): 1278-1284.

Lewis, N. J.; Hurnik, J. F. 1998. The effect of some common management practices on the ease of handling of dairy cows. Applied Animal Behaviour Science, 58: 3-4.

Munksgaard, L.; de Passille, A. M.; Rushen, J.; Herskin, M. S.; Kristensen, A. M. 2001. Applied Animal Behaviour Science, 73: 15-26.

Munksgaard L. A.; de Passille A. M. B.; Rushen J. B.; Ladewig J. 1999. Dairy cows' use of colour cues to discriminate between people. Applied Animal Behaviour Science, 65: 1-11.

Munksgaard, L.; de Passille, A. M.; Rushen, J.; Thodberg, K.; Jensen, M. B. 1997. Discrimination of people by dairy cows based on handling. Journal of Dairy Science, 80: 1106-1112.

Nakao, T.; Sato, T.; Moriyoshi, M.; Kawata, K. 1994. Plasma cortisol response in dairy cows to vaginoscopy, genital palpation per rectum and artificial insemination. Journal of Veterinary Medicine, 41: 16-21.

Pajor, E. A; Rushen J.; De Passille A. M. B. 2003. Dairy cattle's choice of handling treatments in a Y-maze. Applied Animal Behaviour Science, 80: 93-107.

Pinheiro Machado Fo, L. C. M.; Hötzel, M. J.; Yunes, M. C.; Silveira, M. C. A. C.; Furnaletto, G.; Lenzi, A. 2001. Is there a relationship between fear of humans and social rank in Holstein cows? Proceedings of the 35th International Congress of the ISAE, Davis, USA, p.65.

Rushen, J.; de Passille, A. M. B.; Munksgaard, L. 1999a. Fear of people by cows and effects on milk yield, behavior, and heart rate at milking. Journal of Dairy Science, 82 (4): 720-727.

Rushen, J.; Taylor, A. A.; de Passille, A. M. 1999b. Domestic animals' fear of humans and its effect on their welfare. Applied Animal Behaviour Science, 65 (3): 285-303.

Waiblinger S.; Menke C.; Korff, J.; Bucher, A. 2004. Previous handling and gentle interactions affect behaviour and heart rate of dairy cows during a veterinary procedure. Applied Animal Behaviour Science, 85: 31-42.

Yunes, M. C. 2001. Efeito da hierarquia social na produção, na reprodução e na interação humano-animal de vacas leiteiras. Dissertação de Mestrado, Universidade Federal de Santa Catarina, Brasil, 93pp. 